\title{
Get a clear view on glaucoma: the BMO-MRW way
}

\section{Victor Koh}

\section{Department of Ophthalmology, National University Hospital, Singapore}

The association between glaucoma and myopia has been well established and there are multiple population-based studies supporting this association. The mechanisms explaining this association are still unclear and evidence seems to point towards the mechanical stretching of the posterior segment of the eye. This poses another diagnostic challenge to ophthalmologists, as the structural alteration of the optic nerve head $(\mathrm{ONH})$ morphology in myopic eyes may mimic glaucomatous damage. Variations in the morphology include but are not limited to optic disc tilting, torsion, peripapillary atrophy, and enlargement of the optic disc. In addition, the presence of posterior staphyloma may degrade the quality of imaging modalities such as optical coherence tomography (OCT) or colour photographs. This applies to both OCT images of the $\mathrm{ONH}$ and macula. The current study has highlighted the challenges of obtaining good quality, focused, and segmentation error-free OCT scans of myopic eyes with glaucoma. To complicate matters, the aforementioned structural changes to the posterior pole of the eye are associated with visual field defects, which can overlap with glaucoma visual field changes. One of the differentiating features is that myopic visual field changes are less likely to progress compared to glaucoma visual field changes if left untreated.

It is important to recognize that surface landmarks of the optic disc and cup may not accurately represent neuroretinal structure for several reasons. First, the optic disc margin in highly myopic eyes may not be easy to delineate accurately due to variation in $\mathrm{ONH}$ morphology. Second, Chauhan et al. has shown that topographical landmarks may not approximate well with the true anatomical structures below the surface of the $\mathrm{ONH} .{ }^{1}$ In particular, the Bruch's membrane opening position may not coincide with the optic disc margin, which is commonly used in a slit lamp biomicroscopy examination. The deviation could be larger if the $\mathrm{ONH}$ morphology varies in eyes with high myopia. Bruch's membrane is a consistent landmark that is easily identifiable on the cross-sectional OCT imaging of the $\mathrm{ONH}$, which remains stable over time. Third, the variation in ONH morphology makes it challenging to account for the orientation of the neuroretinal rim tissue from surface topography. Bruch's membrane opening-minimum rim width (BMO-MRW) measurements showed better diagnostic performance in detecting early glaucoma compared to peripapillary retina nerve fibre layer (pRNFL). ${ }^{2}$ However, Zheng et al. also showed that in 
highly myopic eyes (axial length of more than $26 \mathrm{~mm}$ ), close to one-third of eyes will have indiscernible Bruch's membrane opening, which will affect the quality of this structural imaging modality. ${ }^{3}$

In this study by Ch'ng et al., ${ }^{4}$ the OCT image quality for BMO-MRW scans was better compared to pRNFL, which is clinically useful to know, especially in myopic eyes with glaucoma. This provides a more reliable trend-based monitoring of an objective measurement for this challenging group of patients. The lower image quality of the pRNFL scan could be attributed to a larger sampling area of the posterior pole resulting in a higher likelihood of a defocused field affected by the posterior staphyloma. After excluding poor quality scans, there was low agreement between PRNFL and BMO-MRW measurements, which again highlighted the unpredictable variation in the posterior pole anatomy of highly myopic eyes.

\section{References}

1. Chauhan BC, Burgoyne CF. From clinical examination of the optic disc to clinical assessment of the optic nerve head: a paradigm change. Am J Ophthalmol. 2013;156(2):218-227.e212.

2. Chauhan BC, O'Leary N, AlMobarakFA, etal. Enhanced detection of open-angle glaucoma with an anatomically accurate optical coherence tomography-derived neuroretinal rim parameter. Ophthalmology. 2013;120(3):535-543.

3. Zheng F, Wu Z, Leung CKS. Detection of Bruch's Membrane Opening in Healthy Individuals and Glaucoma Patients with and without High Myopia. Ophthalmology. 2018;125(10):1537-1546.

4. Ch'ng T, Tan JP, Tassha HA, Farrah BJ, Ahmad BMS. Bruch's membrane opening-minimum rim width and peripapillary retinal nerve fibre layer thickness measurement in myopic eyes with glaucoma. Malaysian Journal of Opthalmology. 2021;3(2):78-90. 